\title{
Water polarization under thermal gradients
}

\author{
Fernando Bresme ${ }^{1,2, *}$ and Anders Lervik ${ }^{3,1}$, Dick Bedeaux ${ }^{2,3}$ and Signe Kjelstrup ${ }^{2,3}$ \\ 1 Department of Chemistry, Imperial College London $S W^{r} 72 A Z$, London UK, \\ 2 Centre for Advanced Study at the Norwegian Academy of Sciences and Letters, Oslo, Norway, \\ ${ }^{3}$ Department of Chemistry, Norwegian University of Science and Technology, Trondheim, Norway
}

(Dated: June 21, 2008)

\begin{abstract}
We investigate the response of bulk liquid water to a temperature gradient using non-equilibrium molecular dynamics simulations. It is shown that the thermal gradient polarizes water in the direction of the gradient, leading to a non-negligible electrostatic field whose origin lies in the water reorientation under non-equilibrium conditions. The dependence of the magnitude of the electrostatic field with the temperature gradient is in agreement with non-equilibrium thermodynamics theory. We conclude that temperature gradients of the order of $10^{-2} \mathrm{~K} / \AA$ could result in fairly large polarizations $\sim 10^{6} \mathrm{~V} / \mathrm{m}$.
\end{abstract}

PACS numbers: 05.70Ln,44.10+i,61.20.Ja,65.20De,66.10.C-

Non-equilibrium phenomena play an essential role in many processes of relevance in biology, physics and materials science. Chemical reactions at surfaces can result in large thermal gradients in small volumes, which may lead to non-equilibrium processes. Thermal gradients often result in a number of coupling effects; mass separation (Soret effect) or or reversible heat conduction conduction (Peltier effect) [1]. The latter provides the physical background for the manufacture of thermoelectric devices [2].

The investigation of non-equilibrium phenomena is becoming a central topic in nanoscale systems. Nowadays, it is possible to induce very large thermal gradients on nanoscopic scales. As a matter of fact, metal nanoparticles can be heated with electromagnetic radiation [3]. This method is expected to result in very large thermal gradients around the nanoparticle $\left(10^{8} \mathrm{~K} / \mathrm{m}\right)$ [4]. These large thermal gradients may induce phase transformations when the nanoparticles are in contact with materials [3], and are strong enough to destroy biological cells. This notion is currently being exploited in medical applications concerned with cancer therapy [5]. These investigations are posing many fundamental questions regarding the mechanism of heat transfer at the nanoscale.

Non-equilibrium thermodynamics (NET) provides the theoretical formalism to describe the response of materials and biomolecules to external gradients [1]. More importantly, it provides a background to understand the coupling effects that often appear under non-equilibrium conditions. One of such effects appears when an isotropic polarizable material is subjected to a thermal gradient. NET predicts that the materials will polarize as a response to the gradient. There are several examples in the literature of related effects, where a temperature gradient can induce molecular orientation. It has been shown that liquid crystals can reorient as a response to heat conduction [7]. Lehmann [8] showed, shortly after the discovery

*Electronic address: f.bresme@imperial.ac.uk of liquid crystals, that the average molecular orientation of a cholesteric liquid crystal shows a uniform rotation under the effect of a temperature gradient.

One may wonder whether such coupling effects between molecular orientation and temperature gradients may be observed in other systems. In this article, we discuss such effect for the most important solvent in biology and materials science, water. Water reorientation under a thermal gradient has not been discussed before to the best of our knowledge. We show that thermal gradients achievable in experiments, can induce water reorientation and as a consequence large polarization fields. We start our discussion with the non-equilibrium thermodynamics background of this particular thermoelectric effect.

Let us consider an isotropic polarizable medium subjected to a heat flux. According to non-equilibrium thermodynamics the entropy production is given by $[1,9]$

$$
\sigma=-\frac{1}{T} \frac{\partial \mathbf{P}}{\partial t} \cdot\left(\mathbf{E}_{\mathbf{e q}}-\mathbf{E}\right)-\frac{1}{T^{2}} \mathbf{J}_{\mathbf{q}} \cdot \nabla T
$$

where, $\mathbf{P}$ is the polarization, $\mathbf{E}_{\mathbf{e q}}=\mathbf{P} /\left(\chi_{e} \varepsilon_{0}\right)$ is the equilibrium electric field, $\chi_{e}=\epsilon_{r}-1$ is the electric susceptibility, $\epsilon_{r}$ the relative dielectric constant, and $\varepsilon_{0}$ the vacuum permittivity. The symbol $\mathbf{E}$ represents the electrostatic field and $T$ is the temperature at a given point in the sample. The dot - indicates a contraction of two vectors. The resulting linear flux-force relations for this system are given by,

$$
\begin{aligned}
\frac{\partial \mathbf{P}}{\partial t} & =-\frac{L_{p p}}{T}\left(\mathbf{E}_{\mathbf{e q}}-\mathbf{E}\right)-\frac{L_{p q}}{T^{2}} \nabla T \\
\mathbf{J}_{\mathbf{q}} & =-\frac{L_{q p}}{T}\left(\mathbf{E}_{\mathbf{e q}}-\mathbf{E}\right)-\frac{L_{q q}}{T^{2}} \nabla T
\end{aligned}
$$

The first equation gives the displacement current, $\partial \mathbf{P} / \partial t$, and the second gives the heat flux, $\mathbf{J}_{\mathbf{q}}$, in terms of the driving forces $-\left(\mathbf{E}_{\mathbf{e q}}-\mathbf{E}\right) / T$ and $-\nabla T / T^{2}$ and the Onsager coefficients $L_{i j}$, which satisfy the Onsager reciprocal relation $L_{p q}=L_{q p}$.

We are interested in the water response in the stationary state. In that case the polarization is timeindependent, $\partial \mathbf{P} / \partial t=0$. Moreover, at the stationary 
state and considering that there are not free charges in the system $\mathbf{P}=-\varepsilon_{0} \mathbf{E}$, and it is straightforward to derive a relationship between the electric field and the thermal gradient,

$$
\mathbf{E}=-\left(1-\frac{1}{\epsilon_{r}}\right)\left(\mathbf{E}_{\mathbf{e q}}-\mathbf{E}\right)=\left(1-\frac{1}{\epsilon_{r}}\right) \frac{L_{p q}}{L_{p p}} \frac{\nabla T}{T}
$$

Equation (4) shows that the electrostatic field originating from the polarization of the sample is proportional to the temperature gradient. The electric field induced by the temperature gradient should be larger for materials with high dielectric constant. The dielectric constant of liquid water is high, $\epsilon_{r} \approx 78$, making such a polarization most likely. To test this hypothesis we have performed non-equilibrium molecular dynamics simulations of water using a realistic model. We note that the presence of ions in water would modify the dielectric effect we are investigating here, because the thermal polarization will compete with the water polarization induced by the salt. This situation is relevant to Seebeck effects in electrolyte solutions [10-13].

Non-equilibrium molecular dynamics simulations of water under a thermal gradient were performed using a method originally developed in reference [14], which has been extended by Bresme et al. [15, 16] to investigate charged systems. Two regions are defined in the simulation box, one at the edges of the simulation box, which acts as a source of heat, and another one in the center of the simulation box, which acts as a sink of heat. The temperature gradient is generated by rescaling the velocities of the molecules in these two regions, ensuring that the total momentum of the system is conserved. This method generates a heat flux, while the pressure along the simulation box is constant. Hence non equilibrium simulations offer a route to compute the equation of state of a substance for a specific isobar. This idea has been used to test the hypothesis of local equilibrium $[13,15]$.

Water was modeled using the Central Force Model (CFM). The CFM includes molecular flexibility, enabling changes in the water dipole moment as a response to the environment. The functional form of the CFM was originally proposed by Lemberg and Stillinger [17], and it has been revised recently. We consider here the version proposed by one us (FB) $[16,18]$. We have shown that the modified CFM reproduces many of the thermodynamic and dynamic properties of water in a wide range of temperatures. It accurately describes the dielectric properties of water $[16,18]$, the predicted dielectric constant is $\varepsilon_{r}=82$, in excellent agreement with the experimental result, $\varepsilon_{r}=78.3$. Full details on the Central Force Model and the simulation set-up can be found in reference [16]. The main difference with respect to those simulations is that in this work we have employed the Wolf's method [19, 20], instead of the Ewald method, to compute the electrostatic interactions. The Ewald method can introduce artificial effects in the computation of the electrostatic interactions of polarized systems, such as the ones considered here. These artificial effects result from the interactions of the dipoles in the simulation box and their replicas. Wolf's method avoids this problem, because it truncates the coulombic interactions ensuring charge neutralization inside the cutoff volume.

The simulated system consisted of 1280-3240 water molecules arranged in a prismatic box with dimensions, $\left\{L_{x}, L_{y}, L_{z}\right\}=\{5,1,1\} \times L_{z}$ or $\{10,1,1\} \times L_{z}$, with $L_{z}=19.73 \AA$ or $L_{z}=14.79 \AA$ respectively. The temperature gradient was imposed along the the $x$-direction. A typical simulation consisted of $1.4 \mathrm{~ns}$ of production time with a simulation time step of $\delta t=3 \times 10^{-16}$ s. To ensure that our calculations corresponded to the stationary state we computed the internal energy flux profile, $J_{u}$, along the simulation box. The simulation box was divided in layers, $l$, perpendicular to the $x$-axis. For each layer we computed the internal energy flux through,

$$
\begin{aligned}
& \mathbf{J}_{u}(l)=\frac{1}{2 V} \sum_{i=1}^{N \in l} m_{i}\left(\mathbf{v}_{i}-\mathbf{v}\right)^{2}\left(\mathbf{v}_{i}-\mathbf{v}\right)+ \\
& \frac{1}{V} \sum_{i=1}^{N \in l} \phi_{i}\left(\mathbf{v}_{i}-\mathbf{v}\right)-\frac{1}{2 V} \sum_{i=1}^{N \in l} \sum_{j \neq i}^{N}\left(\left(\mathbf{v}_{i}-\mathbf{v}\right) \cdot \mathbf{F}_{i j}\right) \mathbf{r}_{i j}
\end{aligned}
$$

where $m_{i}$ and $\mathbf{v}_{\mathbf{i}}$ are the mass and the velocity of particle $i, \mathbf{v}$ is the barycentric velocity of the system, $\phi_{i}$ is the potential energy of particle $i$ and $\mathbf{F}_{i j}$ is the force acting between particles $i$ and $j$. To calculate the profiles of the internal energy flux and other quantities discussed below, we divided the simulation box in $N_{l}=120$ layers. The volume of each layer, $V$ in eqn. (5), is $V=\left(L_{x} / N_{l}\right) \times L_{y} \times$ $L_{z}$. In the absence of mass flux, the internal energy flux should be equal to the imposed heat flux, $\mathbf{J}_{q}=\mathbf{J}_{u}$, which can be obtained from the following continuity equation,

$$
\mathbf{J}_{q}=\left\{\frac{ \pm \Delta u}{2 \delta t A}, 0,0\right\}
$$

where $\Delta u$ is the average kinetic energy added to (' + ' sign in eqn. (6)) or removed from ('-' sign), the hot and cold layers, respectively. Figure 1 shows the internal energy flux profile for one of the systems investigated in this work. The internal energy flux (eqn. (5)) away from the cold and hot thermostatting layers is constant and equal to the imposed heat flux (eqn. (6)). This result shows that our simulations have reached the stationary state. In the following we discuss the water response to the thermal gradient. We have performed simulations of liquid water for a thermodynamic state away from coexistence conditions. In this way we avoided crossing the liquid-vapor coexistence line and the formation of liquid-vapor interfaces. Figure 2 shows the equation of state for liquid water for two non-equilibrium simulations with temperature gradients $\left\{T_{H}, T_{C}\right\}=(475,325) \mathrm{K}$ and $(450,350) \mathrm{K}$ (simulation box $\{5,1,1\})$, which correspond to heat fluxes, $2.6 \times 10^{10}$ and $1.4 \times 10^{10} \mathrm{~W} / \mathrm{m}^{2}$ respectively. The pressure for both simulations is $\mathrm{P}=1.4 \mathrm{kbar}$ 


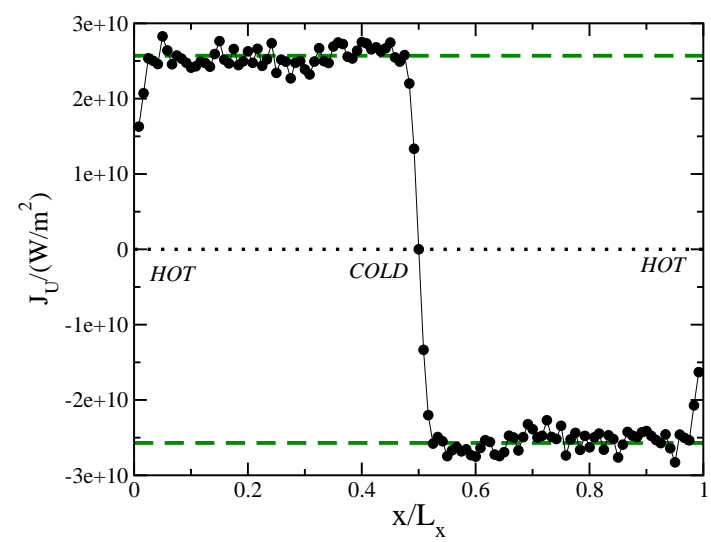

FIG. 1: Heat flux profile for $\left(T_{H O T}, T_{C O L D}\right)=(475,325) \mathrm{K}$, corresponding to a heat flux $J_{q}=2.6 \times 10^{10} \mathrm{~W} / \mathrm{m}^{2}$. HOT and $C O L D$ indicate the position of the thermostats, dashed horizontal lines represent the imposed flux (eqn. (6)), and the symbols the flux computed from eqn. (5).

within statistical uncertainty. The simulated equation of state shows excellent agreement with available equations of stated fitted to experimental results [21]. We note that the water structure of the thermodynamic states investigated here is slightly distorted with respect to the characteristic hydrogen bond structure of the liquid at ambient temperature (cf. Fig. 2).

We have quantified the water polarization along the direction of the thermal gradient by computing the electric field,

$$
E_{x}(x)=\frac{1}{\varepsilon_{0}} \int_{-\infty}^{x} \rho_{q}\left(x^{\prime}\right) d x^{\prime}
$$

where $\rho_{q}\left(x^{\prime}\right)$ represents the charge density defined as, $\rho_{q}\left(x^{\prime}\right)=q_{O} \rho_{O}\left(x^{\prime}\right)+q_{H} \rho_{H}\left(x^{\prime}\right)$, where $q_{i} \rho_{i}\left(x^{\prime}\right)$ is the number of charges per unit volume of species $i$ at position $x^{\prime}$. The polarization along the simulation box is given by $\mathbf{P}(x)=-\nabla \rho_{q}(x)=-\varepsilon_{0} \mathbf{E}(x)$.

The main result of this work is reported in figure 3 . We show that the thermal gradient does indeed polarize water. Due to our simulation set-up, two fields of opposite sign develop around the cold layer. The sign of the field indicates that the water molecules reorient with the dipole pointing toward the cold layer. The oscillatory behavior of the field is a consequence of the finite size of the simulation box and the boundary conditions imposed by the hot and cold layers (cf. Fig. 3). Elongating the simulation box reduces the oscillatory behavior, and the field shows almost a constant value that is compatible with the functionality predicted by NET (c.f. eqn (4)).

The electric field induced by the thermal gradient is very significant, $\sim 10^{8} \mathrm{~V} / \mathrm{m}$, for a characteristic gradient of $\sim 10^{10} \mathrm{~K} / \mathrm{m}$. Such electric fields are typical of biological membranes and ionic thin films [22]. We find that the linear dependence between the electrostatic field and the temperature gradient, predicted by non-equilibrium thermodynamics theory (c.f. eqn. (4)), is fulfilled in our

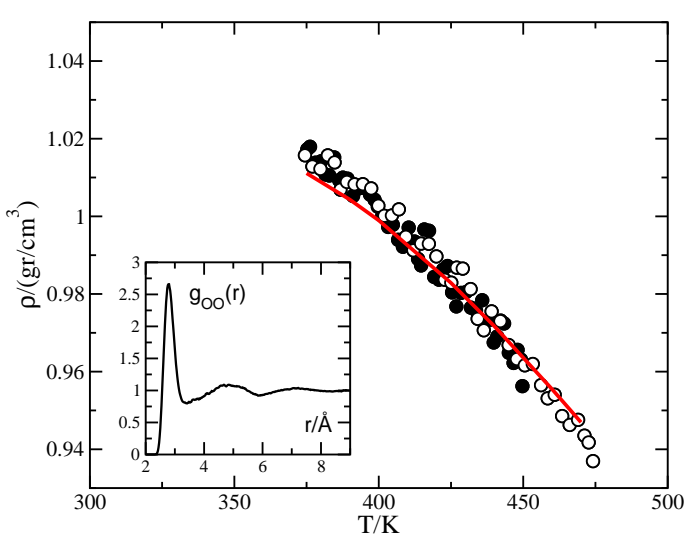

FIG. 2: Equation of state of liquid water for the isobar, $P=1.4$ kbar investigated in this work. Symbols are results from non equilibrium simulations corresponding to heat fluxes, $2.6 \times 10^{10}$ (open circles), and $1.4 \times 10^{10} \mathrm{~W} / \mathrm{m}^{2}$ (filled circles). The line is the experimental isobar for water obtained using the Pitzer-Sterner equation of state [21]. The inset shows the oxygen-oxygen pair correlation function of water at this pressure and temperature $T=400 \mathrm{~K}$ (corresponding to the average temperature of the non equilibrium simulations). The coordination number of the first solvation shell is 5 as compared with 4.5 at ambient conditions $(\mathrm{T}=298 \mathrm{~K})$.

simulations. According to NET the fields generated by two different thermal gradients, $\nabla T_{1}$ and $\nabla T_{2}$ are simply related by, $E_{x, 1} / E_{x, 2}=\nabla T_{1} / \nabla T_{2}$. This relationship is fulfilled in our computer simulations (c.f. Fig. 3). We note that in non-equilibrium thermodynamics, the Onsager coefficients in eqn. (4) are independent of the thermal gradient.

The linear relationship between the electric field and the temperature gradient provides a route to estimate the ratio of the Onsager coefficients $L_{p q} / L_{p p}$. From the value of the electrostatic fields and the average temperature of the simulations, $400 \mathrm{~K}$, we estimate that the ratio $L_{p q} / L_{p p}$ is of the order of $5 \mathrm{~V}$. A fitting of the electric field profile to equation (4), assuming that $\left(1-1 / \epsilon_{r}\right) L_{p q} / L_{p p}$ is independent of the local temperature, and using the local temperatures and the temperature gradient obtained from the simulations, gives a similar estimate for $L_{p q} / L_{p p} \approx 8 \mathrm{~V}$, (cf. Fig. 3). Using the estimate $L_{p q} / L_{p p} \approx 5 \mathrm{~V}$, and equation (4) one can predict the magnitude of the electric field that an experimental gradient would induce in water. Heating of metal nanoparticles with electromagnetic radiation may generate thermal gradients of the order of $10^{8} \mathrm{~K} / \mathrm{m}$ [4]. Such gradients could result in electrostatic fields of the order of $10^{6} \mathrm{~V} / \mathrm{m}$. Similarly, recent experiments on biological cells have reported gradients between $\mathrm{Ca}^{2+}$-ATPases and the surrounding solution of the order of $10^{6} \mathrm{~K} / \mathrm{m}$ [23], which would result in fields of about $10^{4} \mathrm{~V} / \mathrm{m}$.

One question that remains to be addressed is why the water molecules reorient in the temperature gradient? This question can be answered considering the system's entropy production. We write the entropy production in 


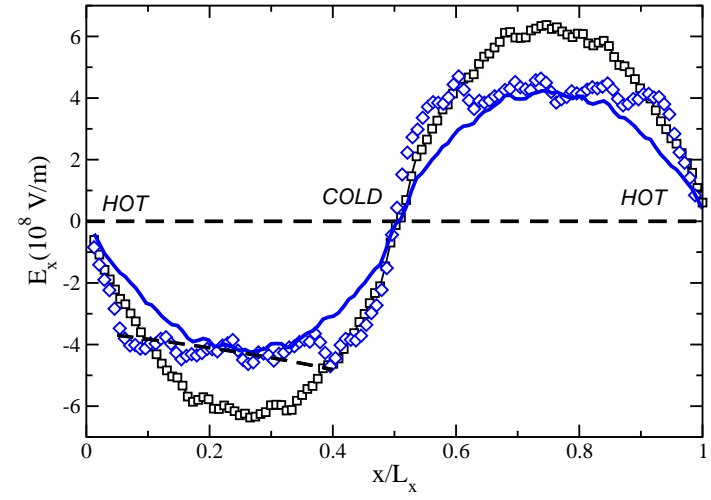

FIG. 3: Electrostatic field along the simulation box. The symbols represent electrostatic fields for different temperature gradients; $J_{q, 1}=2.6 \times 10^{10} \mathrm{~W} / \mathrm{m}^{2}\left(\nabla T_{1}=3 \mathrm{~K} / \AA\right)$ (squares), $J_{q, 2}=1.8 \times 10^{10} \mathrm{~W} / \mathrm{m}^{2}\left(\nabla T_{2}=2 K / \AA\right)($ circles $)$. The full line represents the electrostatic field for $J_{q, 2}$ obtained from $J_{q, 1}$ assuming that eqn. (4) is fulfilled, i.e., $E_{x, 2}=E_{x, 1} \nabla T_{2} / \nabla T_{1}$. The dashed line is a fit of the simulation results to eqn. (4)

a form slightly different to that given in eqn. (1),

$$
\sigma=\left(L_{q q}-\frac{L_{q p} L_{p q}}{L_{p p}}\right)\left|\frac{\nabla T}{T^{2}}\right|^{2}+\frac{1}{L_{p p}}\left|\frac{\partial \mathbf{P}}{\partial t}\right|^{2}
$$

When a thermal gradient is applied to a homogeneous system, a displacement current, is set up, that moves the system to the new state. At stationary conditions $\partial \mathbf{P} / \partial t=0$. The entropy production of the new state decreases with respect to that of the isotropic system if the system gets polarized, because $L_{q p} L_{p q} / L_{p p}=L_{q p}^{2} / L_{p p}>$ 0 . This is the driving force for the polarization of the sample. We note that the coupling between polarization and heat transfer has also an effect on the thermal conductivity. The latter decreases with respect to the unpolarized case. Hence, the polarized system as a whole becomes less effective as a conductor of heat.

The polarization induced by a temperature gradient is a reversible process. We have shown that a temperature gradient can result in large electric fields. Considering the reciprocal relations one can conclude that a displacement current will contribute considerably to the heat flux across the system. A ratio $L_{p q} / L_{p p}$ of $\sim 5 \mathrm{~V}$ gives a heat flux contribution of about $50 \mathrm{~kJ} / \mathrm{m}^{2} \mathrm{~s}$ for a displacement current of $1 \mathrm{~A} / \mathrm{cm}^{2}$. This suggests that the effect discussed here could be observed by measuring the heat flux in a sample subjected to an oscillating electrostatic field.

We thank the Centre for Advanced Study at the Norwegian Academy of Science and Letters for financial support. FB would also like to thank the Royal Society. AL would like to thank NTNU for a scholarship to visit Imperial College. The simulations were performed at the Imperial College High Performance Computing Service.
[1] S.R. de Groot and P. Mazur, Non Equilibrium Thermodynamics, North-Holland - Amsterdam (1962).

[2] G. Jeffrey Snyder and E.S. Toberer, Nature Materials, 7 105 (2008)

[3] H.H. Richardson et. al., Nano Lett., 6783 (2006).

[4] A.O. Govorov et. al. Nanoscale Res. Lett. 184 (2006).

[5] P.K. Jain, I.H. El-Sayed abd M.A. El-Sayed, NanoToday 218 (2007).

[6] R. Hołist and M. Litniewski, Phys. Rev. Lett. 100055701 (2008).

[7] G.W. Stewart, D.O. Holland and L.M. Reynolds, Phys. Rev. 58174 (1940).

[8] O Lehmann, Ann. Phys., (Leipzig) 2649 (1900).

[9] M. Marvan, Czech, J. Phys B, 191240 (1969).

[10] J.N. Agar, Thermogalavanic Cells in: Delahay and Tobias, Advances in Electrochemistry and Electrochemical Engineering, 3, Wiley (1963).

[11] B.R. Brown, M.E. Hughes, C. Russo, Phys. Rev. E, 70 031917 (2004).

[12] H.H. Girault, J. Chem. Soc. Faraday Trans. I, 842147 (1988).
[13] S. Kjelstrup and D. Bedeaux, Non-equilibrium thermodynamics for heterogeneous systems Series on Statistical Mechanics, Vol. 16, World Scientific, Singapore (2008).

[14] T. Ikeshoji and B. Hafskjold, Molec. Phys. 81251 (1994).

[15] F. Bresme, B. Hafskjold and I. Wold, J. Phys. Chem. 1001879 (1996).

[16] F. Bresme, J. Chem. Phys. 1157564 (2001).

[17] H. L. Lemberg and F. H. Stillinger, J. Chem. Phys. 62 1677 (1975).

[18] F. Bresme, J. Chem. Phys. 1084505 (1998).

[19] D. Wolf, P. Keblinski, S.R. Phillpot, and J. Eggebrecht, J. Chem. Phys. 1108254 (1999).

[20] C.J. Fennel, J. Daniel Gezelter, J. Chem. Phys. 124 234104 (2006).

[21] K.S. Pitzer and S.M. Sterner, J. Chem. Phys 1013112 (1994).

[22] J. Faraudo and F. Bresme, Phys. Rev. Lett., 92236102 (2004).

[23] M. Suzuki, V. Tseeb, K. Oyama and S. Ishiwata, Biophys. J.: Biophys. Lett., L46 (2007). 